\title{
Relative contrast in a-Si and c-Si in ADF-STEM imaging
}

\author{
Z. Yu*, D. A. Muller ${ }^{* *}$ and J. Silcox ${ }^{* * *}$ \\ * Physics Department, Cornell University, Ithaca, NY 14853 \\ ** Bell Laboratories, Lucent Technologies, Murray Hill, NJ 07974 \\ *** School of Applied and Engineering Physics, Cornell University, Ithaca, NY 14853
}

Z-contrast ADF images of crystals are sensitive to crystal orientation [1] whereas ADF STEM images of isotropic amorphous material should be invariant under orientation changes. Thus an interface between amorphous material and crystalline material should show variations in contrast depending on orientation. One simple question arises naturally: which side of the interface looks brighter in an ADF-STEM image? The answer is not as straightforward as might be expected and may cause some confusion. In this paper we clarify this question by presenting an experimental example to show the high sensitivity of ADFSTEM contrast to sample orientation. A tilt of just a few degrees reverses the intensity contrast across an amorphous silicon/ crystal silicon interface. The effect is very noticeable in images of silicon transistors where the crystalline silicon channel and polycrystalline gate electrode often have different intensities, complicating quantitative measures of the critical gate oxide thickness measurements.

A cross-section TEM sample of an interface of crystal silicon and amorphous silicon was prepared by typical tripod polishing and was studied with a $200 \mathrm{kV}$ JEOL 2010F TEM/STEM at Bell Labs. The microscope is equipped with two ADF detectors: a low angle ADF (LAADF) detector extends from 20 to 64 mrad and a high angle ADF (HAADF) detector extends from 64 to $200 \mathrm{mrad}$. The probe forming aperture semi-angle was set to $7.5 \mathrm{mrad}$ to ensure the LAADF signal remained incoherent $\left(\theta_{\mathrm{LA}} \sim 3 \theta_{0}\right)$. The sample is first oriented so that the crystal side is on the (110) zone axis. Then the sample is tilted away from the zone axis about the $<001>$ direction (the direction perpendicular to the interface in the plane of the sample (see Figs. 1 and 2)). At each tilt position, a LAADF image and a HAADF image are recorded simultaneously.

Figure 1 shows a tilt series of ADF images taken at low magnification with the sample tilted away from the (110) zone axis at angles of $0^{\circ}, 1^{\circ}, 2^{\circ}, 4^{\circ}$ and $6^{\circ}$, respectively. Upon tilting, the intensity of the amorphous side stays essentially constant in both the LAADF image and the HAADF image, reflecting the facts that amorphous material is structurally isotropic and that the sample thickness stays essentially unchanged for a tilt of a few degrees. When the sample is at $0^{\circ}$ tilt the crystal side is much brighter than the amorphous side in both the LAADF and the HAADF images. This is because strong channeling peaks form along atomic columns inside zone-axis crystal and as a result more electrons are scattered to the ADF detectors $[1,2]$. When the sample is tilted away from the crystal zone axis, the LAADF and HAADF tilt series exhibit qualitatively different trends. The crystal HAADF intensity decreases significantly at $1^{\circ}$ tilt and becomes comparable to the amorphous signal. At further tilt the HAADF intensities from both regions remain similar to each other. Since the main contribution to the HAADF signal comes from channeling peaks, this corresponds to decreases in the channeling peaks inside a tilted crystal [1]. In contrast, the LAADF intensity in the crystal side increases dramatically at $1^{\circ}$ tilt. This results from some low order diffraction disks that previously fell in the central hole of the LAADF detector at $0^{\circ}$ tilt now reaching the LAADF detector as a result of tilt. However, the LAADF intensity decreases monotonically at further tilt due to the weakening of channeling peaks. At $4^{\circ}$ tilt, the LAADF crystal intensity becomes similar to the amorphous intensity and at $6^{\circ}$ tilt, contrast reversal occurs in the LAADF image: the amorphous side becomes brighter than the crystal side.

Figure 2 shows the corresponding high-magnification tilt series. Similar trends in intensity variations upon tilting as in Figure 1 are seen here as well. The two-dimensional (110) Si lattice is well resolved at $0^{\circ}$ tilt in both the LAADF and HAADF images. When the sample is tilted away in the $<001>$ direction, the HAADF lattice image disappears more quickly than the LAADF image, which retains a weak lattice even at $4^{\circ}$ tilt. Another notable feature of the LAADF image at $0^{\circ}$ tilt is a bright band at the interface (top left images in Figure 1 and 2). This bright band is due to the existence of strain field at the interface [3,4]. As it likely results from a preferential dechanneling [4] due to increased disorder, the bright band totally disappears upon tilting, as the channeling contribution to the signal is also reduced [5].

\section{References:}


[1] R. F. Loane, E. J. Kirkland and J. Silcox, Acta Cryst, A44, 912 (1988).

[2] S. Hillyard, R. F. Loane and J. Silcox, Ultramicroscopy 49, 14 (1993).

[3] G. Duscher et al., Characterization and Metrology for ULSI Technology (1998), p.191.

[4] Z. Yu, D.A. Muller, F. Baumann, J. Silcox, "Contrast effect of strain field in ADF-STEM imaging", Microscopy and Microanalysis 2001 (Springer), p.186.

[5] This work was supported by the Cornell Center for Materials Research (CCMR), a Materials Research Science and Engineering Center of the National Science Foundation (DMR-0079992).
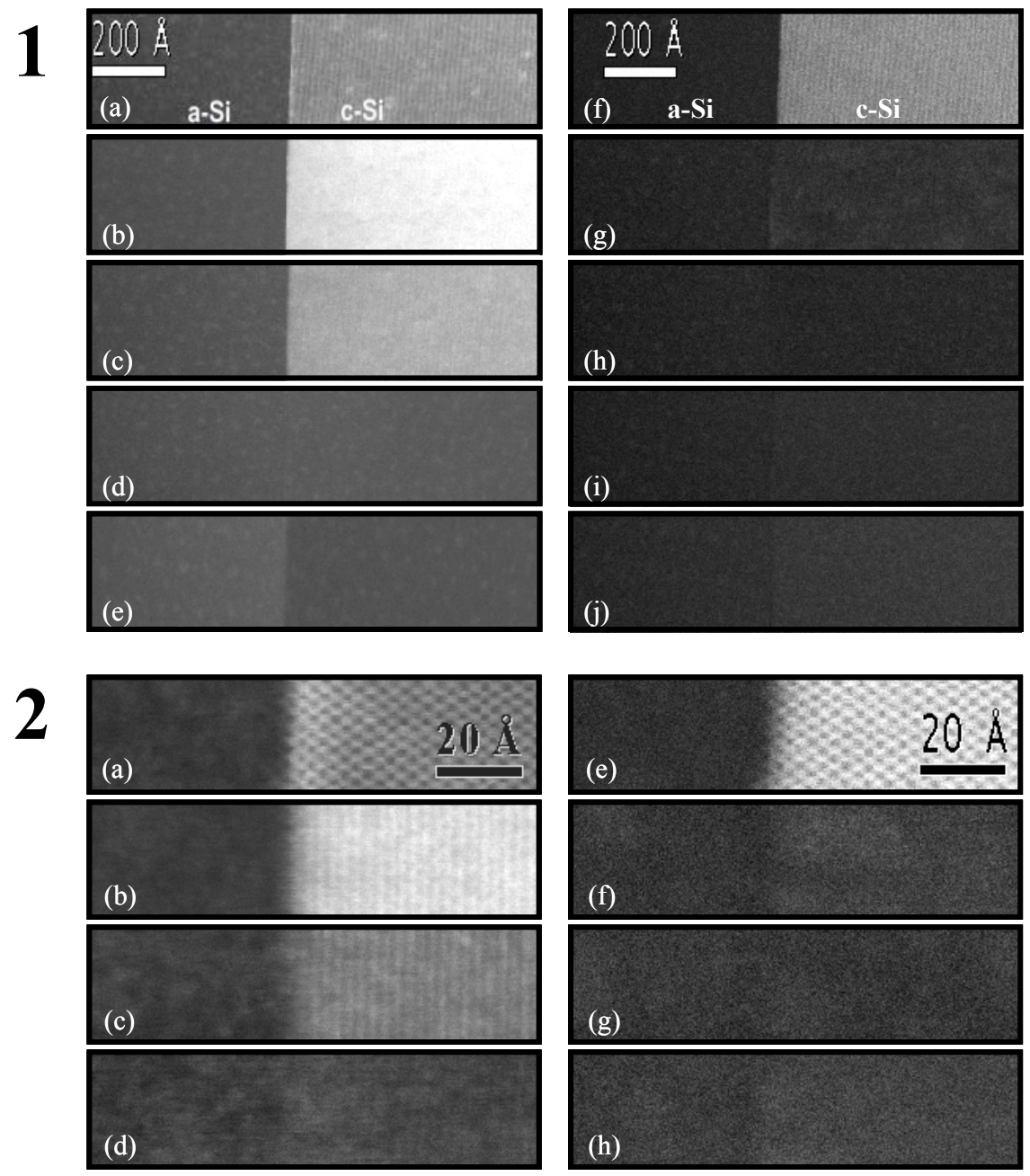

FIG. 1. Low magnification ADF images of c-Si/a-Si interface taken at various tilt positions. The column on the left is the LAADF images and the column on the right the HAADF images. From top to bottom, each pair is taken simultaneously with the sample tilted away from the (110) zone axis at an angle of $0^{\circ}, 1^{\circ}, 2^{\circ}, 4^{\circ}$ and $6^{\mathrm{o}}$, respectively.

FIG. 2. High-resolution ADF images of c-Si/a-Si interface taken at various tilt positions. The column on the left is the LAADF images and the column on the right the HAADF images. From top to bottom, each pair is taken simultaneously with the sample tilted away from the (110) zone axis at an angle of $0^{\circ}, 1^{\circ}, 2^{\circ}$ and $4^{\circ}$, respectively. 